\title{
Contributing Factors to Corneal Deformation in Air Puff Measurements
}

\author{
Sabine Kling and Susana Marcos \\ Instituto de Óptica "Daza de Valdés," Madrid, Spain
}

Correspondence: Sabine Kling, Instituto de Óptica "Daza de Valdés," Calle de Serrano 121, 28006 Madrid, Spain;

sabine@io.cfmac.csic.es.

Submitted: May 30, 2013

Accepted: June 24, 2013

Citation: Kling S, Marcos S. Contributing factors to corneal deformation in air puff measurements. Invest $O p h$ thalmol Vis Sci. 2013;54:5078-5085. DOI:10.1167/iovs.13-12509
Purpose. Air puff systems have been presented recently to measure corneal biomechanical properties in vivo. In our study we tested the influence of several factors on corneal deformation to an air puff: IOP, corneal rigidity, dehydration, presence of sclera, and in vivo versus in vitro conditions.

Methods. We used 14 freshly enucleated porcine eyes and five human donor eyes for in vitro experiments; nine human eyes were used for in vivo experiments. Corneal deformation was studied as a function of: IOP ranging from 15 to $45 \mathrm{~mm} \mathrm{Hg}$ (in vitro); dehydration after riboflavin-dextran instillation (in vitro); corneal rigidity after standard ultraviolet (UV) corneal crosslinking (CXL, in vitro); boundary conditions, that is effect of the presence of the sclera (comparing corneal buttons and whole globes in vitro in pigs); and effect of ocular muscles (comparing human whole globes in vitro and in vivo). The temporal corneal deformation was characterized by the apex indentation across time, the maximal indentation depth, and the temporal symmetry (comparing inward versus outward deformation). The spatial corneal profile was characterized by the peak distance at maximal deformation.

Results. Temporal and spatial deformation profiles were very sensitive to the IOP $(P<0.001)$. The sclera slightly affected the temporal symmetry, while the ocular muscles drastically changed the amount of corneal recovery. CXL produced a significant $(P=0.001)$ reduction of the cornea indentation (by a factor of 1.41), and a change in the temporal symmetry of the corneal deformation profile (by a factor of 1.65), indicating a change in the viscoelastic properties with treatment.

Conclusions. Corneal deformation following an air puff allows the measurement of dynamic properties, which are essential for the characterization of corneal biomechanics.

Keywords: air puff, corneal dynamics, cross-linking, in vivo, in vitro
$T^{\mathrm{s}}$ he use of an air puff to produce a deformation of the cornea is used frequently in ophthalmology to measure the IOP. In applanation tonometry, the IOP is inferred from the force required to flatten (applanate) a constant area of the cornea, making assumptions regarding the contribution of the corneal thickness on the measurements. ${ }^{1,2}$ Typically, in standard applanation tonometry, the force on the cornea is applied by a probe in contact with the cornea. In contrast, air puff noncontact tonometers ${ }^{3}$ use a rapid air pulse to flatten the cornea. Recently, air puff systems have been proposed as potential tools to determine corneal biomechanical properties, taking advantage of the fact that corneal deformation depends on its thickness (from which tonometry measurements must be corrected) and on the corneal biomechanical response. The Ocular Response Analyzer ${ }^{4}$ uses an air puff to deform the cornea into a slight concavity, and monitors the pressures at which the cornea flattens inward and outward, correlating this difference with corneal hysteresis. The availability of highspeed imaging instruments has allowed to capture crosssections of the cornea in real-time during an air puff event. Dorronsoro et al. used a high-speed spectral optical coherence tomography (OCT) system in synchronization with an air puff, and provided seven parameters (amplitude, diameter and volume of the maximum deformation, as well as duration and speed of the increasing deformation period, and the recovery period) that described the dynamics of corneal deformation. ${ }^{5}$ An increase in corneal rigidity by ultraviolet (UV) corneal crosslinking (CXL) in pig corneas (under controlled IOP) resulted in a smaller corneal deformation by the air puff. Recently, a commercial instrument based on Scheimpflug imaging ${ }^{6}$ has been released (Corvis; Oculus, Wetzler, Germany), which captures the corneal dynamic deformation at a speed of approximately $4400 \mathrm{images} / \mathrm{s}$, and extracts the temporal apex deformation, its velocity, and the applanation length from the temporal and spatial profiles of corneal deformation. The breadth of information extracted from the direct view of the corneal deformation holds promise to yield clinically relevant parameters correlated with the biomechanical properties. The possibility of measuring corneal stiffness in vivo would allow an early diagnosis of certain pathologies (such as keratoconus, which weakens the corneal tissue $e^{7,8}$ ), as well as the quantification of the success of treatments that alter the corneal stiffness (such as CXL, used increasingly to stiffen the corneal tissue and to halt keratoconic progression ${ }^{9}$ ). Only few other nondestructive systems to measure the corneal biomechanical properties in vivo have been proposed: ultrasound techniques, ${ }^{10}$ which require eye immersion, and Brillouin microscopy, ${ }^{11}$ a new emerging noncontact technique that allows determining the corneal static elasticity modulus with high spatial resolution, although in its present form it cannot access 
the dynamic properties of the cornea. According to studies on corneal biomechanics, ${ }^{12}$ the cornea is a viscoelastic tissue and, hence, its relaxation time would be an interesting factor to be measured along with corneal elasticity.

Measuring corneal deformation following an air puff, therefore, is a promising technique to measure the dynamic biomechanical properties of the cornea. However, corneal deformation also depends on other geometric and structural factors besides the inherent mechanical properties of tissue. This is relevant particularly to interpret the in vivo measurements provided by corneal air puff instruments. In our study, we evaluated the contribution of different parameters to the corneal deformation by an air puff: The influence of IOP (by performing experiments in vitro at different IOPs), the role of corneal thickness and corneal dehydration (by comparing the deformation of virgin corneas to that of corneas treated with a dextran-containing solution), the role of corneal rigidity (by comparing the deformation of nontreated corneas with corneas treated with $\mathrm{CXL}$ ), the role of corneal boundaries (absence/presence of the sclera, by comparing the deformation of corneal buttons attached to an artificial chamber and deformation of whole eyes), and the role of the ocular muscles and other in vivo/in vitro potential differences in tissue properties (by comparing the deformation in vitro and in vivo). The information provided by the experiments allowed us to identify the parameters that best relate to the corneal biomechanical properties.

\section{MeTHODS}

Air puff corneal imaging was performed in pig and human corneas and eyes, in vitro and in vivo measurements under different conditions.

\section{Air Puff System}

A recently Food and Drug Administration (FDA)-approved air puff system (Corvis; Oculus) was used for the experiments. The Corvis system is composed of an air compressor emitting a controlled air puff on demand. Similar to the Ocular Response Analyzer and other noncontact tonometers, the air puff has a Gaussian shape with a peak pressure of approximately $110 \mathrm{~mm}$ $\mathrm{Hg}$ (D. Luce, CR; Reichert, Inc., Buffalo, NY). In the Corvis system, the release of the air puff is synchronized with an ultrafast Scheimpflug camera that captures 140 corneal images during the air puff event (lasting approximately $20 \mathrm{~ms}$ ). For the measurement, the eye is positioned in front of the system at a distance of $11 \mathrm{~mm}$ between the corneal apex and the air tube. When the eye is aligned and the Scheimpflug image is in focus, the air puff gets released automatically and the cornea imaged during the deformation event. Alternatively, the air puff also can be emitted manually. As the entire measurement happens faster than the blink reflex, in vivo measurements are not affected by motion.

\section{Conditions and Experimental Protocols}

Measurements were performed in pig and human corneas, under different conditions of pressure, dehydration, corneal stiffness, and boundary (fixation) conditions.

Pig Eyes. A total of 14 fresh enucleated pig ( 6 months old) eyes was obtained from a local slaughterhouse (before scalding) and used within 4 hours postmortem.

Human Eyes. Donor eyes were obtained through an agreement between the Fundación Universidad Autónoma de Madrid and Instituto de Óptica (CSIC). Human patients were normal volunteers, and signed an informed consent after receiving an explanation regarding the nature of the study. All protocols followed the tenets of the Declaration of Helsinki and had been approved by the Institutional Review Boards. In vivo air puff measurements were performed on 9 eyes of 7 healthy human subjects aged $35.4 \pm 10.7$ years. In vitro measurements were performed on 5 enucleated donor eyes $(82.6 \pm 4.5$ years $)$.

Experimental Protocols. Measurement conditions and different data sets are summarized in Table 1.

Part 1-Different IOPs (Data Set 1). A set of 5 in vitro virgin porcine whole eye globes was used to assess the impact of IOP on the corneal deformation measurement. The eyes were mounted in a customized holder preventing global motion during the air puff ejection. Then, the IOP was increased from 15 to $45 \mathrm{~mm} \mathrm{Hg}$ and five air puff measurements were taken in each step. For the IOP adjustment an automated pressure system (NE-500; New Era Pump Systems, Inc., Wantagh, NY) was used that has been described previously. ${ }^{13}$ Briefly, a needle was introduced through the optical nerve head connecting the eye over a syringe to a water column. Custom routines written in Matlab (Mathworks, Natick, MA) then were used to control a motor moving a syringe to change the height of the water column. Simultaneously, the pressure in the system (and, hence, the IOP) was recorded by a pressure sensor (part of the NE-500; New Era Pump Systems, Inc.) for accurate regulation.

Part 2-Corneal Thickness and Hydration State (Data Set 1). The eyes from Part 1 subsequently were used in an experiment to assess the impact of corneal thickness and hydration state, produced by riboflavin-dextran solution. ${ }^{14}$ IOP was set to $15 \mathrm{~mm} \mathrm{Hg}$. In this experiment, the first two stages of standard CXL treatment ${ }^{15}$ were performed: (1) de-epithelialization and (2) application of $0.125 \%$-riboflavin-20\%-dextran solution for 30 minutes. This included a prior removal of the epithelium. As in Part 1, the IOP was varied from 15 to $45 \mathrm{~mm}$ $\mathrm{Hg}$ and five air puff measurements were taken in each IOP step.

Part 3-Corneal Rigidity (Data Set 1). The eyes from Part 2 subsequently were used to assess the impact on corneal rigidity, produced CXL. ${ }^{13}$ The IOP was adjusted to $15 \mathrm{~mm} \mathrm{Hg}$, and the last stage of the CXL treatment was performed: Illumination with $367 \mathrm{~nm}$ UV-light and $3 \mathrm{~mW} / \mathrm{cm}^{2}$ for 30 minutes. As in Parts 1 and 2, the IOP was varied from 15 to 45 $\mathrm{mm} \mathrm{Hg}$ and five measurements were taken at each IOP step.

Part 4-Corneal Buttons Versus Whole Eye Globes (Data Set 2). A new set of nine porcine eyes in vitro was used to compare corneal deformation in whole globes and corneal buttons, which allowed investigating the contribution of the sclera to the overall deformation.

Whole eye globes were mounted in a customized holder preventing global motion during the air puff ejection. The IOP was set to $15 \mathrm{~mm} \mathrm{Hg}$ and five air puff measurements were taken on each eye. Then, the corneas were excised and mounted in an artificial anterior chamber. The internal

Table 1. Overview on the Different Data Sets, Treatment, and Conditions Used in the Experiments

\begin{tabular}{llccc}
\hline Data Set & \multicolumn{1}{c}{ Treatment } & CCT, $\boldsymbol{\mu m}$ & $\begin{array}{c}\text { Number } \\
\text { of Eyes }\end{array}$ & Condition \\
\hline 1 & Virgin, pig & 898 & 5 & In vitro \\
& Riboflavin-Dextran, pig & 586 & & \\
& Cross-linking, pig & 211 & & \\
2 & Globe virgin, pig & 878 & 9 & In vitro \\
3 & Button virgin, pig & 993 & & \\
& Virgin, human & 551 & 9 & In vivo \\
& Virgin, human & 768 & 5 & In vitro \\
\hline
\end{tabular}


pressure was set to $15 \mathrm{~mm} \mathrm{Hg}$ (same as the IOP before) and five air puff measurements were on each cornea.

Part 5-In Vitro Versus In Vivo (Data Set 3). The in vitro experiments in five human donor eyes allowed for comparing the deformation observed in human and porcine eyes. In vivo experiments were performed on nine eyes with normal IOP. Comparing corneal deformation in vivo and in vitro allowed us to determine the contribution of ocular muscles to the overall deformation, and of the potential physiologic differences in vivo/in vitro in the investigations on enucleated eyes. In vitro human whole eyes were mounted in a customized holder (similar to the one used in porcine eyes, but scaled to fit human eye globe dimensions). IOP was set to $15 \mathrm{~mm} \mathrm{Hg}$. Five measurements were obtained in this condition per eye. In vivo measurements were obtained following typical clinical protocols. One measurement was obtained per eye.

\section{Analysis}

The corneal profile for each image captured during the deformation event was exported from the Corvis software (Corvis ST; Oculus). Corneal apex indentation as a function of time and the corneal profile at maximal deformation were analyzed. The following parameters were obtained from these deformation data:

1. Peak-to-peak distance $\left(P D_{\max }\right)$, that is, the lateral distance between the two convex peaks in the corneal profile at maximal deformation (Fig. 1A).

2. Temporal symmetry factor $\left(T_{\text {sym }}\right)$, which describes the degree of symmetry of the temporal corneal apex deformation (Fig. 1B). $T_{\text {sym }}$ is the ratio of the two areas under the curve separated by $T_{2}$ and can be calculated from:

$$
T_{s y m}=\frac{\sum_{T_{1}}^{T_{2}} \Delta Y_{\text {apex }}}{\sum_{T_{2}}^{T_{3}} \Delta Y_{\text {apex }}}
$$

where $T_{1}$ is the time where the deformation starts, $T_{2}$ is the time at maximal deformation, and $T_{3}$ is the time when the
A

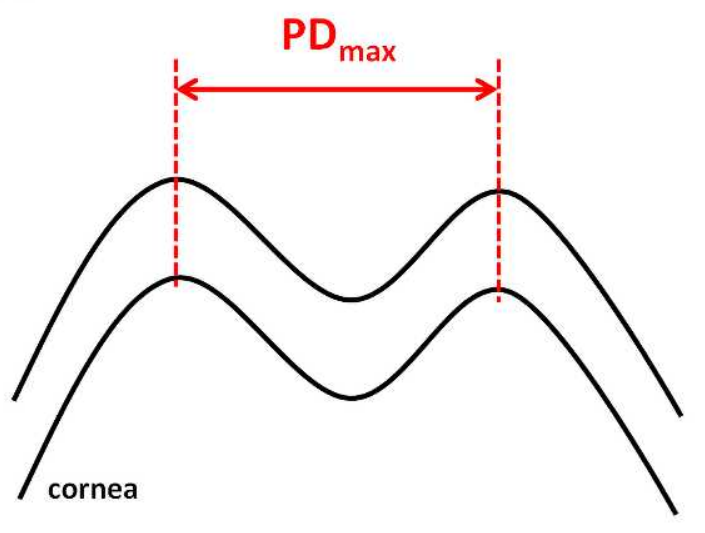

direct air puff response finishes. $\Delta Y_{\text {apex }}$ is the indentation of the corneal apex recorded by the Corvis system (Oculus). $T_{s y m}$ indicates the degree of temporal symmetry in the deformation $\left(T_{\text {sym }}=1\right.$ means a purely axis-symmetric temporal deformation).

3. Direct air puff response, which describes the corneal deformation as a direct response to the applied air puff, that is as long as the air puff applies a force onto the cornea (indicated by time points $T_{1}$ and $T_{3}$ ).

4. Indirect air puff response was defined as the remaining deformation after $T_{3}$, that is after the direct air puff response. It depends on the viscoelastic properties and, for the in vivo condition, it depends additionally on the damping due to the ocular muscles.

5. Corneal central thickness (CCT) before the air puff deformation.

Statistical analysis was done using SPSS software (IBM Corporation, Armonk, NY). Multivariate analysis of variance (MANOVA) was performed to test the overall effect of IOP, geometry, and treatment conditions on the corneal deformation. MANOVA with repeated measures was used to test the repeatability of the measurements. One-way ANOVA was performed to test the differences between individual groups. Confidence levels were set to $95 \%$.

\section{Results}

The major dependencies of the geometric deformation parameters for the different evaluated corneal conditions are summarized in Table 2.

\section{Effect of IOP-Data Set 1}

Temporal Apex Deformation. Figure 2A presents the apex deformation of a virgin porcine whole globe across time. The maximal indentation was very sensitive to variations in IOP $(P<0.001)$. In virgin eyes the maximal indentation varied from 1.262 to $0.550 \mathrm{~mm}$ for IOP ranging between 15 and 45 $\mathrm{mm} \mathrm{Hg}$, decreasing at a rate of $-23.9 \mu \mathrm{m} / \mathrm{mm} \mathrm{Hg}$. The temporal symmetry factor $\left(T_{\text {sym }}\right)$ did not change significantly $(P=0.298)$ with IOP.
B

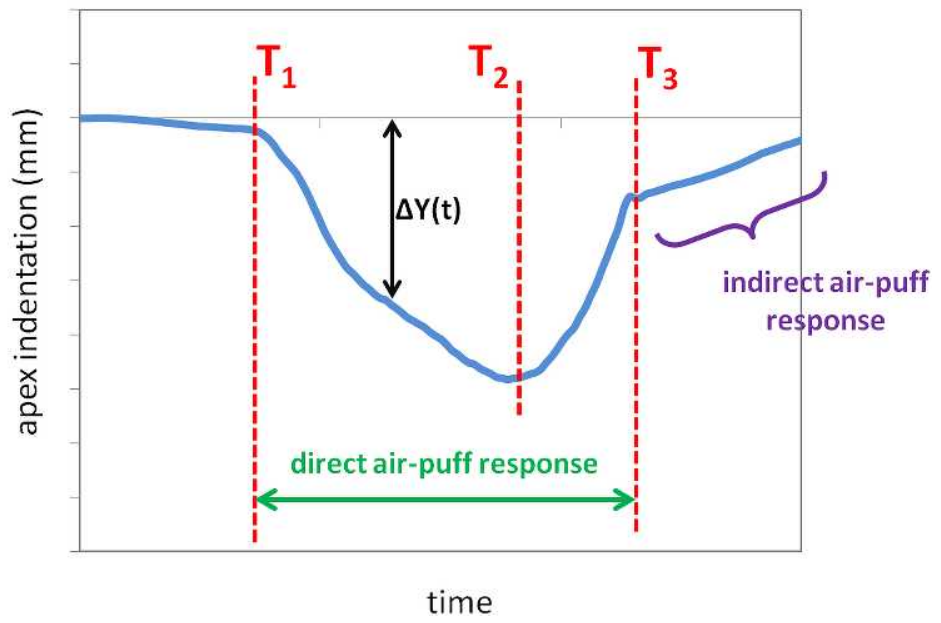

Figure 1. (A) Illustration of the corneal profile at maximal deformation and definition of the $P D_{\max }$. (B) Illustration of the apex indentation as a function of time, and definition of the parameters of the temporal symmetry $\left(T_{s y m}\right)$, direct and indirect air puff response. 
TABLE 2. Summary of the Dependencies of the Geometrical Deformation Parameters for the Different Experimental Conditions

\begin{tabular}{llll}
\hline \multicolumn{1}{c}{ PD } & Maximal Apex Indentation & \multicolumn{1}{c}{$\boldsymbol{T}_{\text {sym }}$} \\
\hline IOP & Linear dependency & Strong, nonlinear dependency & No effect \\
Hydration/CCT & No effect & No effect & No effect \\
Rigidity & Influence & Influence & Influence \\
Button/whole globe & No effect & No effect & Influence \\
In vitro/in vivo & No effect & No effect & No effect \\
\hline
\end{tabular}

Spatial Deformation Profile. The corneal profile at maximal deformation is shown in Figure 2B. For a better comparison the initial apex position was shifted to zero. As expected (and similar to the maximal apex deformation) a strong dependency of the IOP on the maximal distance between peaks $\left(P D_{\max }\right)$ was found $(P<0.001)$.

\section{Effect of Dehydration and CXI-Data Set 1}

Temporal Apex Deformation. Figure 3A shows the different temporal deformation patterns of the corneal apex throughout the CXL treatment. Riboflavin-dextran instillation did not induce a significant $(P=0.978)$ difference in the apex indentation with respect to virgin corneas (virgin, $1.26 \pm$ $0.16 \mathrm{~mm}$; riboflavin-dextran, $1.30 \pm 0.08 \mathrm{~mm}$ ). On the contrary, after CXL we observed a pronounced decrease of the apex indentation (by a factor of 1.67), and change (by factor 1.14) in the symmetry of the temporal apex deformation $\left(T_{\text {sym }}\right)$, both compared to virgin. In general, in virgin corneas and in riboflavin-dextran treated corneas the inward deformation time took longer than the backward deformation (virgin corneas, $T_{\text {sym }}=1.653 \pm 0.25$; riboflavin-dextrantreated corneas, $T_{\text {sym }}=1.463 \pm 0.21$ ). This relation changed after CXL, where the durations of the inward and outward deformation were similar $\left(T_{\text {sym }}=1.002 \pm 0.16\right)$. Differences in the temporal symmetry between virgin and riboflavindextran corneas, and differences in the temporal symmetry between virgin and cross-linked corneas were highly significant $(P<0.001)$.

Spatial Profile Deformation. Figure 3 shows the peakto-peak distances of the corneal profile at maximal deforma-

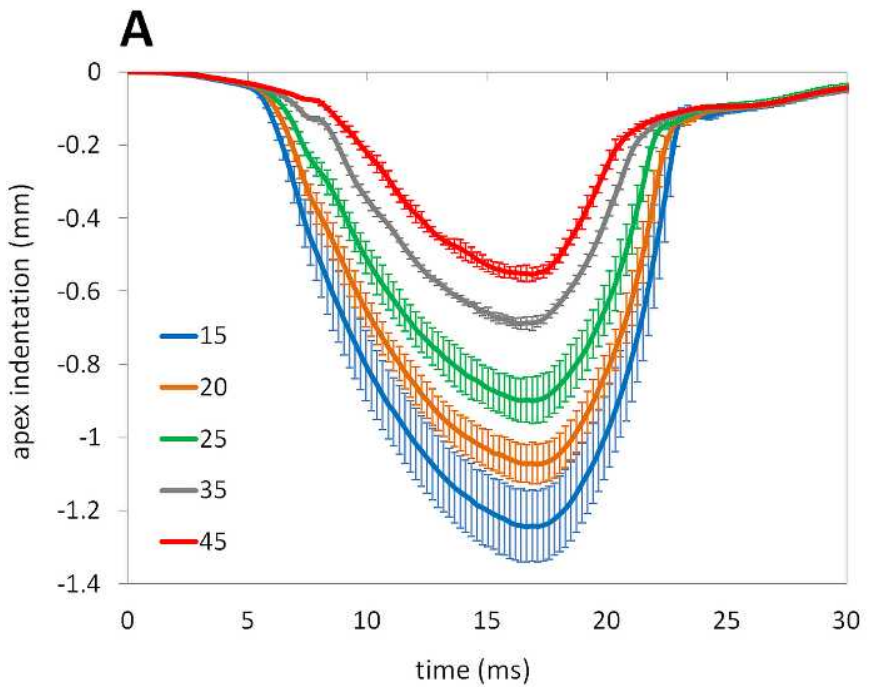
the standard deviation across eyes. porcine cornea), for virgin corneas, riboflavin-dextrantreated corneas, and cross-linked corneas, respectively. No significant difference in the peak distance was observed due to riboflavin-dextran instillation $(P=0.891)$, while CXL produced a highly statistically significant decrease $(P<$ 0.001).

\section{Difference Between Button and Whole Globe- Data Set 2}

Temporal Apex Deformation. Figure 4 shows the apex deformation of in vitro porcine corneas at normal IOP $(15 \mathrm{~mm}$ $\mathrm{Hg}$ ). The maximal indentation depth of the apex (whole globes, $1.221 \pm 0.43 \mathrm{~mm}$; corneal buttons, $1.049 \pm 0.15 \mathrm{~mm}$ ) was not significantly ( $P=0.113$ ) affected by the boundary condition. The deformation of the cornea in whole globes tended to be shorter in time, and the temporal symmetry was significantly $(P=0.002)$ lower (whole globes, $T_{\text {sym }}=1.426 \pm$ 0.16 ; corneal buttons, $\left.T_{\text {sym }}=1.582 \pm 0.17\right)$. The red curves (representing the in vitro porcine eye in Fig. $4 \mathrm{~A}$ and the in vitro human eye in Fig. 5) show the same treatment condition and, hence, differences are due to the interspecies differences between pig and human corneal tissue. Figure $4 \mathrm{~B}$ shows a comparison of air puff corneal deformation in corneal buttons and whole globes following CXL. Similarly, a shorter deformation was observed for whole globes compared to corneal buttons and temporal symmetry was significantly $(P$ $<0.001$ ) different (cross-linked whole globes, $T_{\text {sym }}=1.002 \pm$ 0.16 ; cross-linked corneal buttons, $T_{\text {sym }}=1.309 \pm 0.132$ ).

Spatial Profile Deformation. Maximal peak distance was not significantly different $(P=0.576)$ between whole

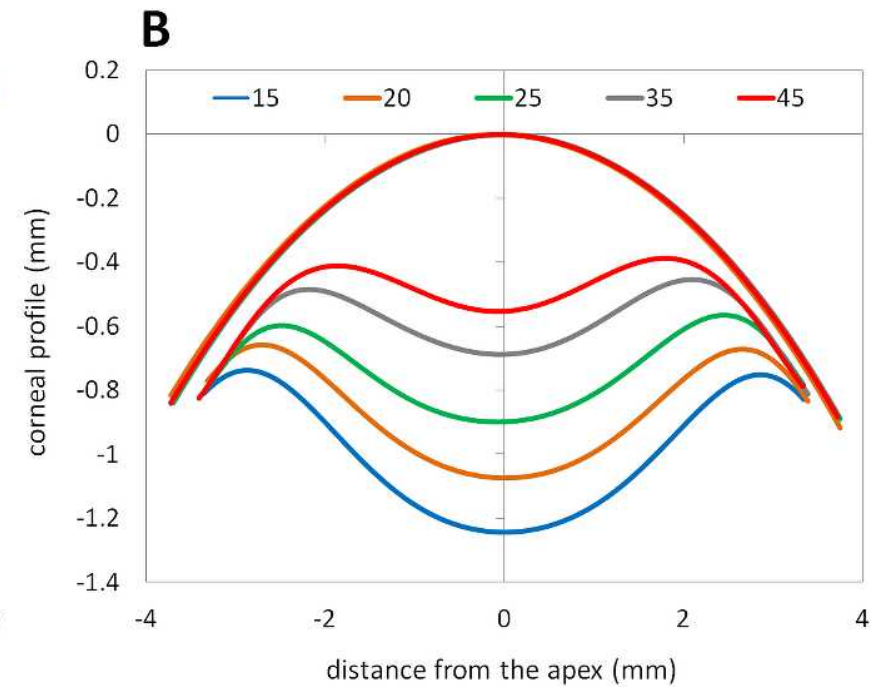

Figure 2. (A) Corneal apex deformation as a function of time. (B) Corneal profile at maximal deformation. Different colors represent different IOPs, ranging from 15 to $45 \mathrm{~mm} \mathrm{Hg}$. Data are for whole porcine eyes. Each point is the average of measurements in five eyes. Error bars stand for 

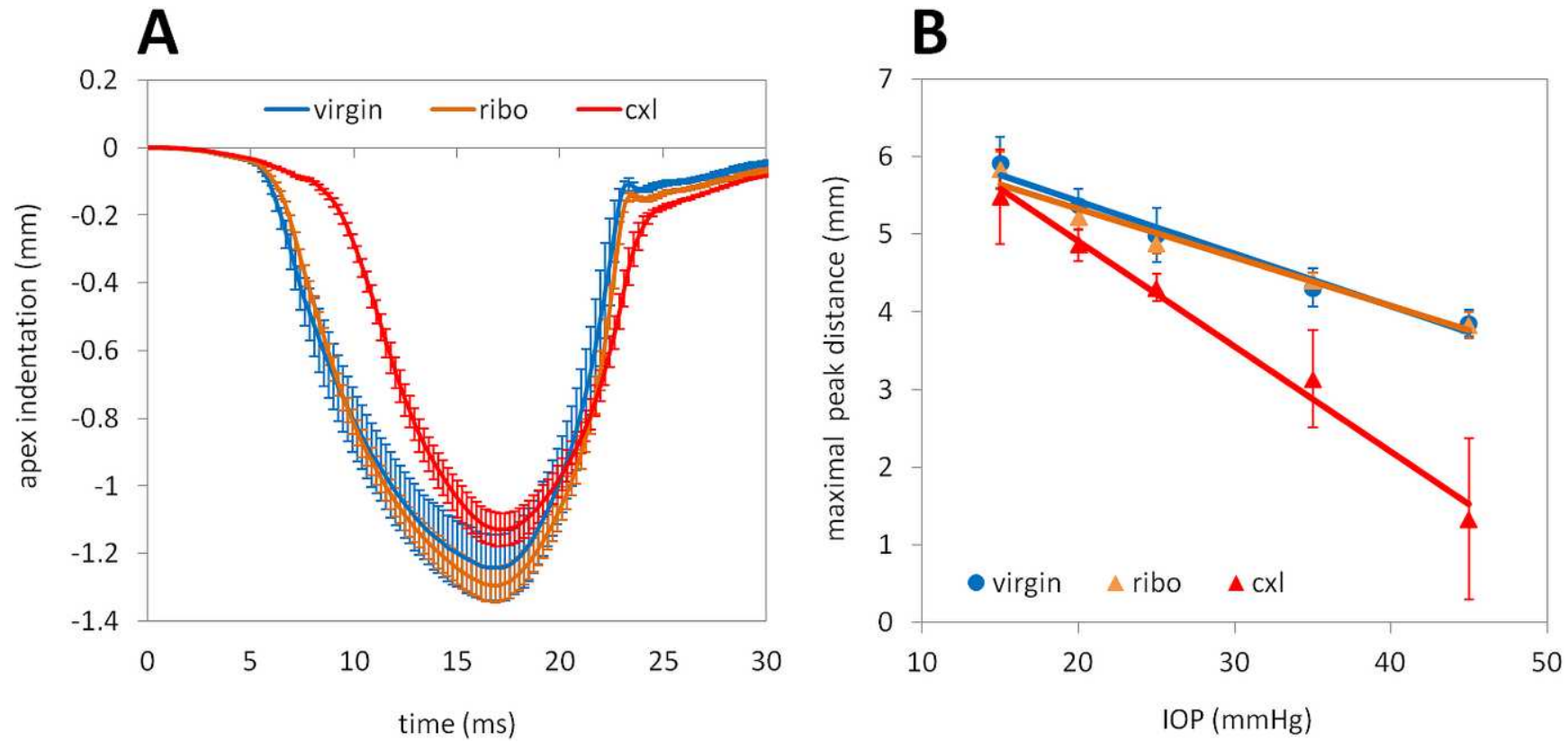

Figure 3. (A) Corneal apex deformation during the deformation event (IOP fixed at $15 \mathrm{~mm} \mathrm{Hg}$ ). (B) Corneal profile $P D_{\max }$ as a function of IOP. Data are average across 5 whole globes each: virgin corneas (blue), riboflavin-dextran-treated corneas (yellow), cross-linked treated corneas (red). Each point is the average of measurements in five eyes.

globe $\left(P D_{\max }=5.57 \pm 0.98 \mathrm{~mm}\right)$ and button $\left(P D_{\max }=5.74\right.$ $\pm 0.39 \mathrm{~mm})$ porcine corneas, indicating a negligible contribution of the sclera in the spatial profile deformation.

\section{Difference Between In Vitro and In Vivo-Data Set 3}

Temporal Apex Deformation. Figure 5 presents the apex deformation of in vivo and in vitro human eyes at normal IOP $(15 \mathrm{~mm} \mathrm{Hg})$. There is a trend for a larger corneal deformation in vivo than in vitro, although the differences are not statistically significant $(P=0.290)$ and could be associated with the age differences between both groups. No significant difference $(P=0.704)$ was found between the temporal symmetry in human whole globes in vitro $\left(T_{\text {sym }}=1.600 \pm\right.$ $0.212)$ and in vivo $\left(T_{\text {sym }}=1.608 \pm 0.264\right)$.

Spatial Profile Deformation. Maximal peak distance was not significantly different $(P=0.384)$ between in vivo human whole globes $\left(P D_{\max }=4.787 \pm 0.18 \mathrm{~mm}\right)$ and in vitro human whole globes $\left(P D_{\max }=4.778 \pm 0.33 \mathrm{~mm}\right)$, indicating a negligible contribution of the ocular muscles in the spatial profile deformation.
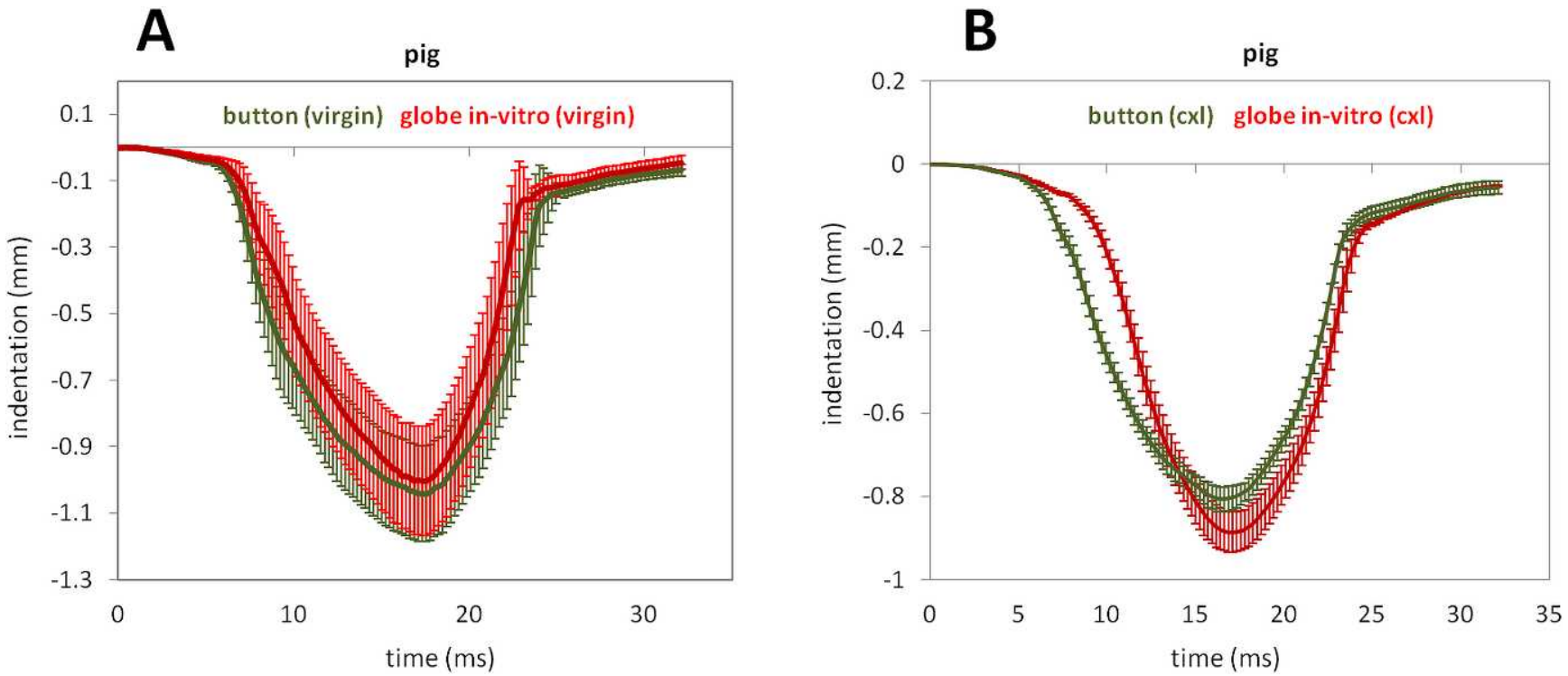

Figure 4. Corneal apex deformation across time in in vitro porcine corneas at IOP $=15 \mathrm{~mm} \mathrm{Hg}$ (whole globes versus corneal buttons). Differences reveal the effect of the sclera. (A) Virgin porcine corneas. Each point is the average of measurements in 9 eyes. (B) Cross-linked porcine corneas. Each point is the average of measurements in five eyes. 


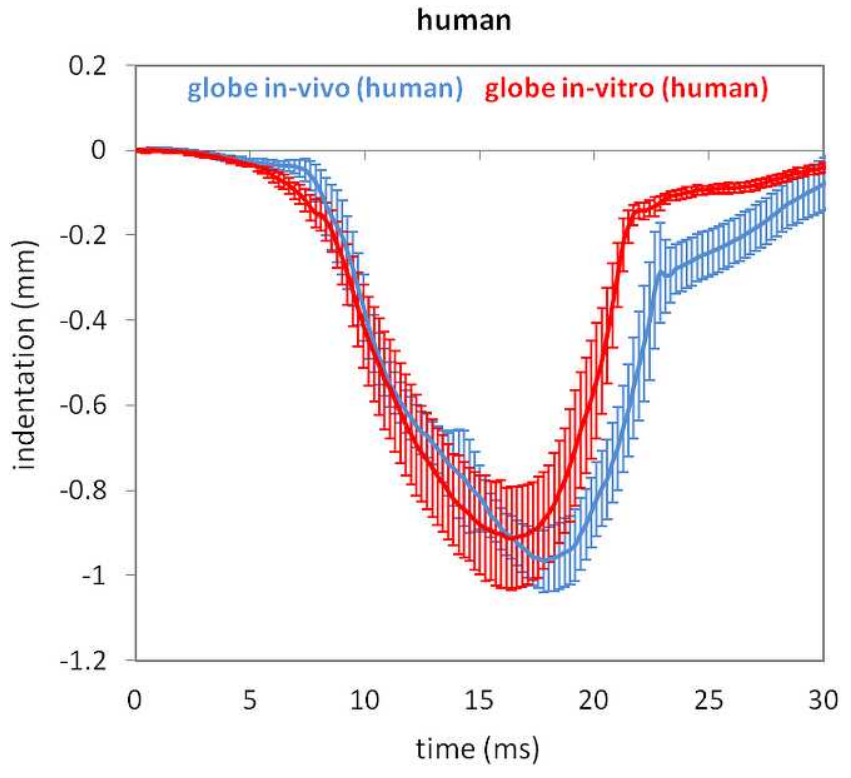

Figure 5. Corneal apex deformation across time in human globes at $\mathrm{IOP}=15 \mathrm{~mm} \mathrm{Hg}$ (in vitro versus in vivo). Each point is the average of measurements in nine eyes in vivo and five eyes in vitro, respectively. Differences reveal the effect of ocular muscles.

\section{CCT-All Data Sets}

Corneal thickness differed largely across the different treatment conditions and data sets (Table 1). Riboflavin-dextran treatment induced a decrease in corneal thickness by $65.3 \%$, which decreased further by $35.9 \%$ after CXL. In vitro virgin human corneas were on average 1.39 times thicker than in vivo human corneas. We also found that corneas in virgin whole globes were $115 \mu \mathrm{m}$ thinner than the contralateral corneas measured as corneal buttons.

\section{Repeatability of Measurements-Data Set 2}

All four analyzed parameters were highly repeatable across the four consecutive measurements. Standard deviations of repeated measurements were maximal apex indentation (whole globe, $\pm 0.064 \mathrm{~mm}$; corneal button, $\pm 0.027 \mathrm{~mm}$ ), maximal peak distance (whole globe, $\pm 0.516 \mathrm{~mm}$; corneal button, $\pm 0.077 \mathrm{~mm}$ ), $T_{\text {sym }}$ (whole globe, \pm 0.102 ; button, \pm 0.045 ), and CCT (whole globe, $\pm 13.3 \mu \mathrm{m}$; corneal button, $\pm 15.4 \mu \mathrm{m}$ ).

\section{Discussion}

The investigation of the effect of different controlled conditions on corneal deformation upon air puff allows a better understanding of the different factors affecting corneal deformation and a better interpretation of these measurements in human patients. The high repeatability of Corvis (Oculus) measurements reported in a recent study was confirmed by our measurements. ${ }^{16}$

We found that corneal deformation following an air puff is highly sensitive to IOP. The amount of apical deformation showed an almost linear correlation with IOP, which confirmed the usefulness as a noncontact tonometer. ${ }^{3}$ It also indicated the need for including IOP as a parameter in the interpretation of the corneal deformation patterns measured in vivo.

In addition, we found that the maximal indentation depth of the apex was correlated with a stiffening of the corneal tissue due to CXL. The fact that riboflavin-dextran-treated corneas experienced a similar deformation to virgin corneas suggested that corneal thinning and geometry changes cancel out the effect of potential biomechanical changes induced by riboflavin-dextran. Thinner corneas generally are expected to deform more; therefore, an increase in corneal density (due to dehydration) could explain the increase in corneal rigidity with dehydration. It is known that dehydration can change the intrinsic biomechanical properties of the corneal tissue. ${ }^{17}$ In our study, we did not find a dependency between corneal deformation and CCT, likely because the observed thickness changes all related to density changes. A recent study suggests that water intake into the cornea decreases its viscosity and cancels out the effect of thickness increase. ${ }^{18}$ Future studies might address the corneal response after partially removing corneal tissue (such as after LASIK or photorefractive keratectomy [PRK] surgery). The dependency of the corneal deformation pattern on geometrical factors would be a limitation in the direct clinical application of air puff deformation parameters to assess corneal biomechanical properties, as it implies the use of correction formulae to isolate the biomechanical contribution from other factors.

We found that the temporal symmetry factor was highly sensitive to the CXL treatment, and depended only slightly on IOP and corneal thickness/dehydration. A change in temporal symmetry suggests an alteration of the viscoelastic material properties (such as the relaxation time). This change in the dynamic behavior of the cornea after CXL can be supported by a recent experimental study, where a uniaxial creep test in cross-linked corneal tissue suggested changes in the viscoelastic properties, in comparison with virgin corneal tissue (Hammer A, et al. IOVS 2013;54:ARVO E-Abstract 4073). Moreover, the delayed start of the apex indentation in crosslinked corneas (compared to virgin or riboflavin-dextran corneas) indicates a higher resistance to deformation due to an increase of corneal stiffness. In our experiments, the sensitivity of the maximal peak distance to CXL increased drastically with higher IOP (Fig. 3B). This effect may be interesting for air puff measurements in glaucoma patients.

The corneal deformation pattern of a whole globe and corneal button showed significant differences in the temporal deformation pattern $\left(T_{s y m}\right)$, whereas no significant difference was observed in the maximal apex indentation and peak distance. As the sclera affected only the temporal apex deformation, the scleral deformation upon air puff must be very small. A minor contribution to the differences between corneal deformation in corneal button and whole globes also may be due to the small, but significant $(P=0.018)$ differences in radii of curvature between the corneal button fixed in the artificial chamber and in whole globes $\left(R_{\text {Button }}=9.18 \pm 0.52\right.$ vs. $\left.R_{\text {Globe }}=8.90 \pm 0.31 \mathrm{~mm}\right)$. The difference observed in corneal thickness between whole globes and corneal button is surprising. Corneal thickness might have increased in buttons as the tissue when cutout is allowed to relax for a short time.

The corneal deformation pattern in in vitro and in vivo human eye globes did not show a significant difference in the maximal peak distance and in the temporal symmetry. The trend (nonsignificant) for a larger corneal deformation in vivo than in vitro could be associated with the age differences between both groups. The most relevant difference was the larger deformation remaining after the direct air puff response in vivo. This observation can be attributed to the contribution of the ocular muscles acting as an external viscoelastic damper, and can be corrected for in the data processing. The addition of the corneal deformation to the damping of the ocular muscles also may explain small differences observed in the maximum corneal apex indentation. Differences are minor despite the great differences in handling and conditions of in vitro and in vivo human corneas. On one hand, the in vitro 
eyes had been stored for up to 24 hours before measurement. This explains the increased corneal thickness due to hydration (Table 1). On the other hand, in vitro eyes were significantly older than in vivo measured eyes, and slight differences in corneal stiffness with aging are expected. ${ }^{19}$

Differences between deformation patterns of porcine and human corneas are consistent with prior knowledge of interspecies corneal biomechanical properties. Porcine corneas deformed to a larger extent and slower than human corneas. This confirms the previously reported difference in viscoelastic behavior between human and porcine cornea: porcine corneas relax more than human corneas (under a given stretch ratio)..$^{20}$

In comparison with the Ocular Response Analyzer (ORA), the corneal response following an air puff was imaged with a higher temporal and spatial resolution in this study, allowing the direct acquisition of corneal deformation patterns. To date, there is no clear evidence that the parameters obtained from the ORA (so-called corneal hysteresis $[\mathrm{CH}]$ and corneal resistance factor $[\mathrm{CRF}]$ ) reveal constant and significant changes with CXL. ${ }^{21,22}$ Although alternative parameters can be extracted from the ORA signal, ${ }^{23}$ the limitation of the system remains that only the temporal change of the slope of the corneal tangent in a small zone is measured. This limitation can be overcome by imaging the entire corneal deformation within a zone of 8 $\mathrm{mm}$ of diameter, which gives access to a higher number of descriptive parameters of the deformation. The high temporal and spatial resolution of the corneal deformation measurements upon air puff ejection, such as those presented in our study, and, in particular, the numerous experimental conditions tested, serve as excellent input data for inverse finite element models, which then will allow retrieving the corneal biomechanical properties (elastic and viscoelastic parameters, i.e., elasticity modules and Prony/ relaxation time constants) and identifying the effect of side factors, such as the IOP, geometry, and boundary conditions.

Other emerging alternatives to measure corneal biomechanical properties in vivo include Brillouin microscopy ${ }^{24}$ and ultrasound, ${ }^{25}$ which primarily address the measurement of the static elastic modulus. Corneal deformation measurements with air puff give access to the dynamic behavior of the corneal tissue and, therefore, reveal different biomechanical properties than the aforementioned systems. Corneal dynamics, including viscoelasticity, have been studied primarily in vitro ${ }^{12,13,20,26}$ and have been addressed only in vivo through the use of the ORA system. ${ }^{21-23}$ In particular, it is expected that corneal changes following CXL treatment affect the viscoelastic properties to a large extent $^{12}$ (Hammer A, et al. IOVS 2013;54:ARVO E-Abstract 4073). Therefore, the information that can be obtained from high resolution imaging of the corneal deformation with air puff will make a valuable contribution in the characterization of the corneal biomechanics in vivo.

In conclusion, the meaningfulness of deformation parameters obtained from the presented air puff measurements depends on their correlation with the biomechanical properties of the corneal tissue. We identified the IOP to be correlated mostly with the maximal indentation depth of the apex, whereas corneal rigidity was identified best by the temporal symmetry factor. The maximal corneal peak distance was correlated with the maximal indentation depth of the apex. The contribution of the ocular muscles acted as an external damper and, when subtracted, the corneal deformation pattern is primarily driven by the real corneal response. Corneal viscoelastic properties are revealed by the temporal symmetry factor and changed after cross-linking.
Our results allowed for a better clinical interpretation of the observed corneal deformation. These data can serve as input data for numerical and analytical models describing the cornea with the aim of predicting surgical outcomes more accurately.

\section{Acknowledgments}

The authors thank Oculus for providing access to the Corvis ST system, and Nicolas Alejandre and Pablo Perez-Merino for their help in the acquisition of human and porcine eyes, respectively.

Supported by Spanish Government FIS2011-25637 (SM) and FPIBES-2009-024560 Predoctoral Fellowship (SK). The research leading to these results has received funding from the European Research Council under the European Union's Seventh Framework Programme (FP7/2007-2013)/ERC Grant Agreement number 294099. The authors alone are responsible for the content and writing of the paper.

Disclosure: S. Kling, None; S. Marcos, Oculus (F)

\section{References}

1. Recep ÖF, Hasiripi H, Nurullah C, Srikatipoglu H. Relation between corneal thickness and intraocular pressure measurement by noncontact and applanation tonometry. J Cataract Refract Surg. 2001;27:1787-1791.

2. Cennamo G, Rosa N, La Rana A, Bianco S, Sebastiani A. Noncontact tonometry in patients that underwent photorefractive keratectomy. Ophthalmologica. 1997;211:341-343.

3. Shields B. The noncontact tonometer. Its value and limitations. Surv Ophthalmol. 1980;24:211-219.

4. Luce DA. Determining in vivo biomechanical properties of the cornea with an ocular response analyzer. $J$ Cataract Refract Surg. 2005;31:156-162.

5. Dorronsoro C, Pascual D, Perez-Merino P, Kling S, Marcos S. Dynamic OCT measurement of corneal deformation by air puff in normal and cross-linked corneas. Biomed Opt Exp. 2012;3: 473-487.

6. Hon Y, Lam A. Corneal deformation measurement using Scheimpflug noncontact tonometry. Optom Vis Sci. 2013;90: $1-8$.

7. Oxlund H, Simonsen AH. Biochemical studies of normal and keratoconus corneas. Acta Opthalmologica. 1985;63:666669.

8. Edmund C. Corneal elasticity and ocular rigidity in normal and keratoconic eyes. Acta Ophthalmol. 1988;66:130-140.

9. Spoerl E, Huhle M, Seiler T. Induction of cross-links in corneal tissue. Exp Eye Res. 1998;66:97-103.

10. Tanter M, Touboul D, Gennisson JL, Bercoff J, Fink M. Highresolution quantitative imaging of cornea elasticity using supersonic shear imaging. IEEE Trans Med Imaging. 2009; 28:1881-1893.

11. Scarcelli G, Pineda R, Yun SH. Brillouin optical microscopy for corneal biomechanics. Invest Ophthalmol Vis Sci. 2012;53: 185-190.

12. Boyce BL, Jones, Nguyen TD, Grazier JM. Stress-controlled viscoelastic tensile response of bovine cornea. J Biomech. 2007; 40:2367-2376.

13. Kling S, Remon L, Perez-Escudero A, Merayo-Lloves J, Marcos S. Corneal biomechanical changes after collagen cross-linking from porcine eye inflation experiments. Invest Ophthalmol Vis Sci. 2010;51:3961-3968.

14. Kling S, Marcos S. Effect of hydration state and storage media on corneal biomechanical response from in vitro inflation tests. J Refract Surg. 2013;29:490-497. 
15. Wollensak G, Spoerl E, Seiler T. Riboflavin/ultraviolet-ainduced collagen crosslinking for the treatment of keratoconus. Am J Ophthalmol. 2003;135:620-627.

16. Hong J, Xu J, Wei A, et al. A new tonometer - the Corvis ST tonometer clinical comparison with noncontact, and goldmann applanation tonometers. Invest Ophthalmol Vis Sci. 2013;54:659-665.

17. Hennighausen H, Feldman ST, Bille JF, McCulloch AD. Anteriorposterior strain variation in normally hydrated and swollen rabbit cornea. Invest Ophthalmol Vis Sci. 1998;39:253-262.

18. Terai N, Raiskup F, Haustein M, Pillunat LE, Spoerl E. Identification of biomechanical properties of the cornea: the Ocular Response Analyzer. Curr Eye Res. 2012;37:553562 .

19. Elsheikh A, Wang D, Brown M, Rama P, Campanelli M, Pye D. Assessment of corneal biomechanical properties and their variation with age. Current Eye Res. 2007;32:11-19.

20. Yanjun Z, Jian Y, Kun H, Zhihui L, Xiuyun L. A comparison of biomechanical properties between human and porcine cornea. J Biomech. 2001;34:533-537.
21. Vinciguerra P, Albe E, Mahmoud AM, Trazza S, Hafezi F, Roberts CJ. Intra- and postoperative variation in ocular response analyzer parameters in keratoconic eyes after corneal crosslinking. J Refract Surg. 2010;26:669-676.

22. Goldich Y, Barkana Y, Morad Y, Hartstein M, Avni I, Zadok D. Can we measure corneal biomechanical changes after collagen cross-linking in eyes with keratoconus? - A pilot study. Cornea. 2009;28:498-502.

23. Spoerl E, Terai N, Scholz F, Raiskup F, Pillunat LE. Detection of biomechanical changes after corneal cross-linking using Ocular Response Analyzer software. J Refract Surg. 2011;27: 452-547.

24. Scarcelli G, Yun SH. In vivo Brillouin optical microscopy of the human eye. Opt Express. 2012;20:9197-9202.

25. Wang H, Prendiville PL, McDonnell PJ, Chang WV. An ultrasound technique for the measurement of the elastic moduli of human conrea. J Biomech. 1996;29:1633-1636.

26. Glass DH, Roberts CJ, Litsky AS, Weber PA. A viscoelastic biomechanical model of the cornea describing the effect of viscosity and elasticity on hysteresis. Invest Ophthalmol Vis Sci. 2008;49:3919-3926. 